\title{
Influence of thermodynamic state on nanojet breakup
}

\author{
H Shin ${ }^{1}$, M Oschwald ${ }^{2}, M M M_{\text {Micci }}{ }^{3}$ and W Yoon ${ }^{1}$ \\ ${ }^{1}$ Department of Mechanical Engineering, Yonsei univ., 134 Sinchon-dong, Seodaemun-gu, Seoul 120-749, Korea \\ ${ }^{2}$ German Aerospace Center (DLR), D-74239, Hardthausen, Germany \\ ${ }^{3}$ Department of Aerospace Engineering, The Pennsylvania State University, 229 Hammond Building University \\ Park, PA 16802-1401 \\ E-mail: burny75@yonsei.ac.kr
}

\begin{abstract}
Using non-equilibrium molecular dynamics, argon nanojet injection was simulated under vacuum conditions. A series of simulations with different shapes of solid platinum injectors were conducted. Observed droplet sizes and jet breakup characteristics resemble the Rayleigh breakup theory. However, the different injector shapes did not cause a significant change in the nanojet breakup behaviour. The liquid temperature inside the injector was found to be a controlling factor in determining the subsequent breakup characteristics. A higher liquid temperature is preferred for the faster nanojet breakup with the shorter breakup length.
\end{abstract}

PACs codes

76D25 Wakes and jets

82C80 Numerical methods (Monte Carlo, series resummation, etc.)

82C24 Interface problems; diffusion-limited aggregation

Submitted to: Nanotechnology

\section{Introduction}

Recently, the need for understanding the mechanics of micro/nano scale behaviour has been emphasized with the remarkable progress in micro and nano machining techniques [1], [2]. In particular, nanojet injection is an interesting research topic in the field of bioengineering because it can deliver a gene or drug directly into biological cells [1], [2]. Moreover, the usage of nanosized injection can be expanded into various scientific fields such as a very fine ink-jet printing technique. However, previous theories and modelling techniques about jet injection have been developed targeting much larger thermodynamic systems with continuum assumptions [3] [4] thus the direct application of these theories to nanoscale systems is uncertain. 
This deviation between a thermodynamic-scale system and a nanoscale system was reported recently from results using carbon nanotubes [5]-[7]. Carbon nanotubes, which are an ideal material to study the unknown physics of nanoscale phenomena revealed a unique behaviour with water in nanotubes. Results from both experiments and molecular dynamics (MD) simulations detected an ordered ice structure of water inside carbon nanotubes with a strong dependency of tube diameter [6], [7]. This liquid-solid transition of confined water occurred at various temperatures that are different than that for bulk liquid. These results emphasize the need of a new starting point for understanding nanosized phenomena. Therefore the numerical technique for predicting nanosized phenomenon should be free from previous assumptions. This explains why MD is becoming popular as a numerical tool for exploring nanoscale physics. MD is a molecular-based method therefore no assumptions are necessary except Newton's $2^{\text {nd }}$ law, thus it is an ideal numerical method for nanosized systems. Non-equilibrium MD is frequently used for predicting the disintegration of a nanojet [8]-[10]. Moseler and Landman showed that the details of nanojet breakup behaviour obtained by MD have significant differences compared to the Navier-Stokes result [8]. Current liquid jet breakup and spray models require many assumptions and experimental correlations those are difficult to obtain in the nanojet [11], [12]. However, MD needs only the correct interatomic or intermolecular potential. Any rigorous equations of state and any formulas for finding thermophysical properties of fluids are unnecessary in MD.

The one obstacle against the feasibility of this prospective numerical technique is a very high computation cost. Basically, MD should calculate all possible intermolecular interactions of every molecule in the system with a time step that should be small enough to capture the rapid molecular motions. This explains why the maximum dimensions in a common MD are nanometer scale.

However, the improvement of computing speed combined with parallel computation techniques places MD near for practical use. Currently the available dimensions in MD are strictly limited to the nanoscale, however considering the developing speed of computer technology it is only a matter of time to be able to enlarge the available system size.

In this study, a MD simulation of argon nanojet injection under a vacuum environment has been investigated with various solid atomic injectors. Due to the vacuum environment, the aerodynamic effect on jet breakup is negligible, thus it is expected that Rayleigh breakup, which is mainly due to the surface tension, would prevail. In addition, the effect of injector geometry on nanojet breakup was examined with two different solid injectors, cylindrical and convergent. Various simulation parameters were examined to determine those that had a significant effect on jet breakup behaviour.

\section{Molecular Dynamics}

The most important choice in MD simulations is the interatomic potential. In the current study, argon is chosen due to its simple structure. Simplicity of argon modelling results in a substantial decrease of 
calculation time with a slightly larger time step. The Lennard-Jones 12-6 potential is used for reproducing argon liquid [13]. A nondimensional form of Lennard-Jones potential is shown in equation 1. Superscript * denotes nondimensional variables.

$$
U^{*}\left(r^{*}\right)=-4\left[\left(r^{*}\right)^{-12}-\left(r^{*}\right)^{-6}\right]
$$

The interaction force $\mathrm{F}^{*}$ between molecular pairs can be directly calculated by the negative spatial derivative of the intermolecular potential function $U^{*}\left(r^{*}\right)$.

$$
\vec{F}^{*}\left(r^{*}\right)=-\vec{\nabla} U^{*}\left(r^{*}\right)
$$

For generating solid injectors, platinum is chosen and the interactions between liquid and solid molecules are predicted by the same Lennard-Jones potential but with different potential parameters. Potential parameters for liquid argon and solid platinum are from reference [14]. These are shown in table 1. The integration of molecular positions and velocities is done by the velocity Verlet algorithm [13].

As mentioned above, there have been several previous attempts to reproduce nanojet injection with MD simulations [8]-[10]. A prominent difference among these works is how to handle the wall effect. There are basically two methods, one is a direct simulation of a solid atomic wall [8] and the other is a diffusive-reflection wall boundary condition [9], [10]. Diffusive reflection can reproduce the no-slip condition at the wall with keeping a desired temperature by a Gaussian thermostat, but it is unable to reproduce the adhesive force between the liquid and the solid. Thus, a solid atomic wall was chosen for our study. The details of the solid modelling technique will be introduced in the following chapter.

Table 1. Basic inter-atomic potential parameters

\begin{tabular}{cccc}
\hline \multicolumn{2}{c}{$\mathrm{Ar}-\mathrm{Ar}$ interaction } & \multicolumn{2}{c}{$\mathrm{Pt}-\mathrm{Ar}$ interaction } \\
\hline mass $_{A r}$ & $6.6318 \times 10^{-26} \mathrm{~kg}$ & mass $_{P t}$ & $3.239 \times 10^{-25} \mathrm{~kg}$ \\
$\sigma_{A r}$ & $3.405 \times 10^{-10} \mathrm{~m}$ & $\sigma_{P t}$ & $3.085 \times 10^{-10} \mathrm{~m}$ \\
$\varepsilon_{A r}$ & $1.6566 \times 10^{-21} \mathrm{~J}$ & $\varepsilon_{P t}$ & $0.894 \times 10^{-21} \mathrm{~J}$ \\
\hline
\end{tabular}

Parallel computation is essential in current MD simulations due to the massive computational cost. Numerical algorithms for parallel computation should be optimized for high calculation efficiency. Basically, there are three algorithms for efficient parallel computation, the spatial, particle and force decomposition methods [15]. Particle decomposition, while easy to code, has large memory and 
communication costs. It is known that spatial decomposition is more efficient than force decomposition with a spatially uniform density condition. However, our target system has a highly non-uniform density distribution so the application of spatial decomposition algorithm causes non-even allocation of calculation load onto each processor. Therefore, the force decomposition has been chosen in our simulation.

As the system size increases, most intermolecular interactions are trivial due to the fast decay of the potential function with the increase of intermolecular distances. Therefore, a way to screen the meaningful interactions from all possible interactions is necessary for better calculation efficiency. For this purpose, we used the Verlet neighbour-list method, which generates the neighbour-lists of every molecule [13]. Once the neighbour-lists are prepared, these lists can be used for tens of time steps depending on a difference between a cut-off radius $r_{c}$ and a larger list radius $r_{1}$. Generally, the process to find neighbour atoms is the most time-consuming part in actual MD simulations, thus the usage of the Verlet neighbour-list method can cause a remarkable improvement on calculation efficiency. The lists of neighbour atoms were calculated every fifty time steps with a $3.5 \sigma$ cut-off radius and a $4.2 \sigma$ list radius, where $\sigma$ is a Lennard-Jones potential parameter for argon. Also, all properties and variables are normalized by units shown in table 2 .

Table 2 Normalizing parameters

\begin{tabular}{cc}
\hline Properties & Normalizing units \\
\hline Length & $\sigma=3.405 \times 10^{-10} \mathrm{~m}$ \\
Force & $\varepsilon / \sigma=4.865 \times 10^{-12} \mathrm{~N}$ \\
Mass & $m=6.6318 \times 10^{-26} \mathrm{~kg}$ \\
Time & $\sigma \sqrt{\mathrm{m} / \varepsilon}=2.154 \mathrm{ps}$ \\
Pressure & $\varepsilon / \sigma^{3}=41.96 \mathrm{MPa}$ \\
Temperature & $\varepsilon / k_{B}=119.98 \mathrm{~K}$ \\
\hline
\end{tabular}

\section{Simulation Setup}

The current calculation can be separated into 3 parts. First is the liquid jet initialization, followed by the production of the solid injector. Then, combining these two, the injection simulation is conducted. The liquid argon jet is initialized with the density from bulk liquid at $P^{*}=0.048$ and $T^{*}=0.917$. Critical 
points of liquid argon are $T_{C}^{*}=1.257$ and $P_{C}^{*}=0.117$. We chose these initial conditions as a representative condition for a stable liquid state. The diameters of the jets, $D^{*}=D / \sigma$, for the convergent and cylindrical injectors were 17.62 and 8.81 respectively. Liquid argon molecules are initially placed in a Face-Centered-Cubic (FCC) lattice then initialized with velocity rescaling [13] until $t^{*}=9.285$. The instantaneous temperature of the atoms in Cartesian coordinate can be calculated from equation 3. $N$ is the number of sampled atoms. $\bar{v}^{*}$ is an average velocity of atoms.

$$
T^{*}\left(t^{*}\right)=\sum_{i=1}^{N} \frac{\left(v_{i x}^{*}-\bar{v}_{x}^{*}\right)^{2}+\left(v_{i y}^{*}-\bar{v}_{y}^{*}\right)^{2}+\left(v_{i z}^{*}-\bar{v}_{z}^{*}\right)^{2}}{3 N}
$$

Velocity rescaling is a direct method to fit the total amount of kinetic energy to our aimed level. This method is shown in equation (4) in nondimensional form.

$$
v_{i}^{*}=\sqrt{\frac{T_{t \arg e t}^{*}}{T^{*}}} v_{i}^{*}
$$

The liquid jet initialization continues until $t^{*}=27.86$ to equilibrate the liquid molecules. Instead of using a solid atomic wall for liquid jet initialization, a diffusive wall condition was used to mimic the wall. Periodic boundary conditions are used at the both ends of the jet [13]. Because the total volume of the system is fixed in this case, we assumed that the absence of a wall adhesive force would not result in a considerable difference compared to an atomic wall. Time step for all the simulations is chosen as $0.929 \times 10^{-3}$ in nondimensional units.

In this study we examined several injectors, both cylindrical and convergent with different convergent angles. Tested convergent angles were 45, 60 and 90 degrees. However, the overall jet breakup behaviour of the different injectors were very similar so the results with the 45 degree injector are chosen as a representative case of all the convergent injector results.

To generate a solid platinum injector, platinum molecules are placed in FCC (111) structure. This structure can be made by the repetition of three hexagonal layers in sequence. All the tested injectors have an axisymmetric shape so the method to produce a solid injector is rather simple. First a solid cube that is slightly larger than the size of the injector is generated with a FCC (111) structure and then the inner and outer radius regions are truncated. The thickness of the solid injector is about 2.5 in nondimensional units. The shapes of the examined injectors are shown in figure 1. The vibrational motion of the solid molecules is simulated by a Hookean spring. A Hookean spring is attached to the original lattice site of each solid molecule so that the solid molecules have a restoring force to their sites. With this restoring force, solid molecules can oscillate from their initial lattice sites while maintaining a constant level of kinetic energy by a velocity rescaling method. Depending on the magnitude of the spring constant, the solid modelling 
method can be called a soft-spring model [16]-[18] or a stiff-spring model [19]. Both models were examined. In the case of the soft-spring model, we assumed the spring constant to be 733.43 , which was equal to the product between the soft-spring constant (150.82) from reference [17] and the mass ratio between platinum and argon atom (4.863). It was chosen as 6000 in the stiff-spring model [19]. However the results from those two models are almost identical so we chose the stiff-spring model for use in the current calculations.

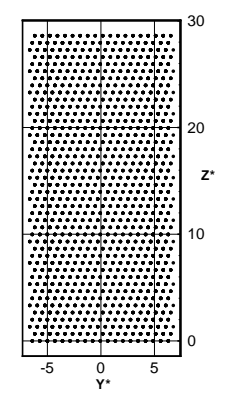

Side View (Y-Z Plane)

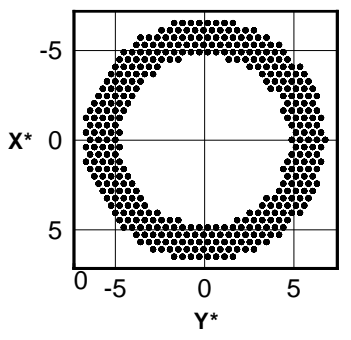

Top View (X-Y Plane)

(a) Cylindrical Injector

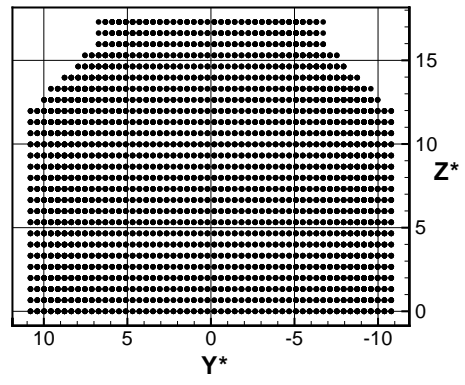

Side View (Y-Z Plane)

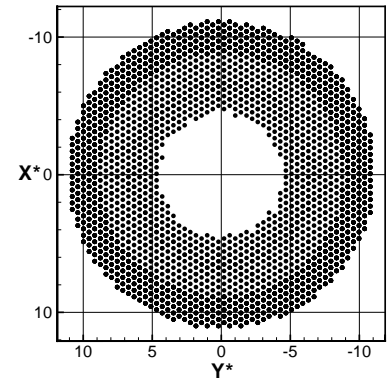

Top View (X-Y Plane)

(b) 45 degree convergent injector

Figure 1. Geometries of tested injectors

Once both the liquid jet and the solid injector have been prepared, the injection simulation is started. Our jet injection method is similar to the pressurized injection method from Moseler et al. [8]. The only difference is that we are pushing liquid jet into the injector with constant speed, not constant pressure. Therefore, it is easier to control the jet injection speed than previous pressurized injection simulations. Our injection method consists of the following sequence. First, the equilibrated liquid jet is frozen to maintain constant interatomic spacing before entering the solid injector. Once the frozen jet enters the solid injector region, the liquid molecules are allowed to move freely with a constant temperature control. Due to the nanoscale jet diameter and high injection speed, the viscous heating from the liquid in the solid injector is overwhelming so constant temperature control is required. Velocity rescaling is also used here as the constant temperature control method [13]. However, the rescaling was made only for the non-axial 
velocities because the direct rescaling of axial velocity may interfere with the development of the axial velocity profile [19]. The non-axial velocity components are rescaled every 50 time steps.

The liquid jet pushing speed was chosen as 1.518 in the cylindrical jet and 0.380 in the convergent jet. The exit cross-section area of the convergent injector becomes 4 times smaller than its inlet area; therefore the average injection speed at the injector exit should be equal to that of the cylindrical jet.

Using the virial theorem [20], the local pressure inside the injector was calculated. The average calculated pressure of the liquid inside the cylindrical injector is approximately 12 nondimensional pressures. Such an extremely high level of pressure results from both high surface friction at the solid wall and high jet injection speed. In previous research from Moseler et al. [8], they used 11.92 (500 MPa) as the injection pressure to reach the steady injection speed of $2.53(400 \mathrm{~m} / \mathrm{sec})$.

In addition, 2-D plotting of various properties of the liquid argon inside the solid injector has been made. General 3-D scatter plots show only the outer shell of surface atoms so the information from the inner atoms is difficult to ascertain. However, 2-D plots can clearly visualize the spatial variation of various liquid properties such as averaged velocity, density, pressure, temperature and surface tension. This reduction of dimension from 3-D to 2-D is made with the assumption of axisymmetric flow. All 2-D and 3-D plots presented here have been sampled during 5,000 time steps so the temporal evolution of the liquid jet can be seen.

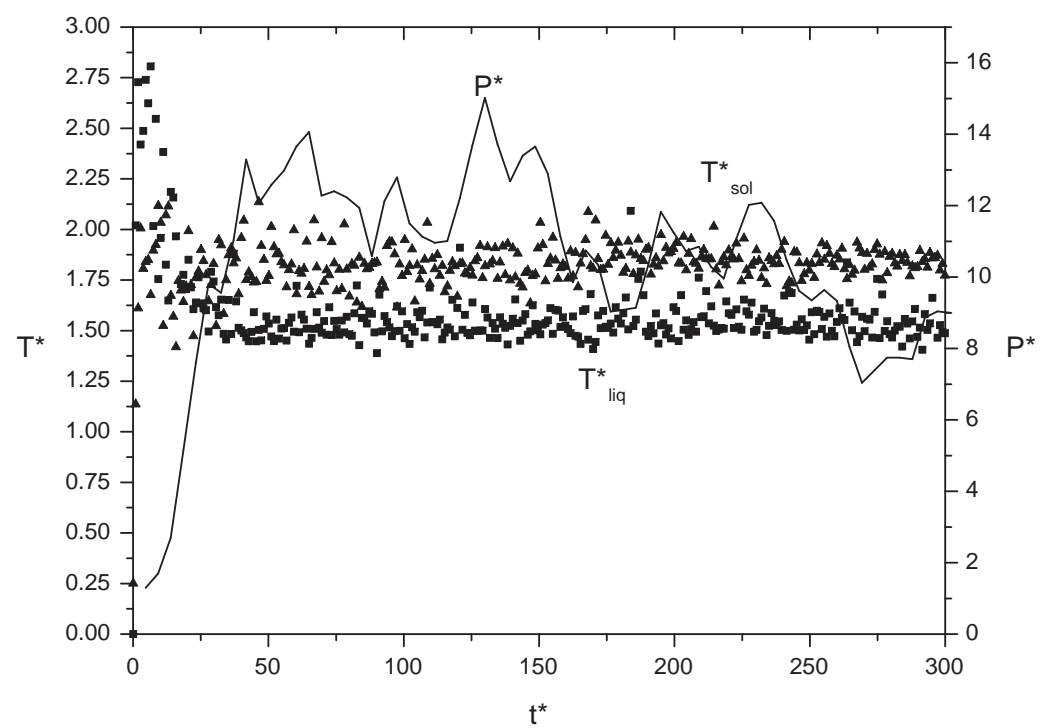

Figure 2. Temporal behaviour of averaged temperatures of solid injector $\left(T_{\text {solid }}^{*}\right)$ and liquid jet $\left(T_{\text {liquid }}^{*}\right)$. Average normalized liquid pressure in the injector is plotted on the right axis. 


\section{Results}

\subsection{Cylindrical injector}

Nanojet injection simulation results with the cylindrical solid injector are discussed in this section. Figures 2 and 3 show the temporal evolution of temperature, pressure and jet breakup behaviour. After the initial transient period, both the liquid inside the injector and the solid wall reach temperatures of 1.50 and 1.83 respectively by velocity rescaling. The measurements of liquid temperature and pressure in figure 2 were made only inside the cylindrical injector region. We chose the solid wall temperature to be slightly higher than the liquid temperature. If the wall temperature is the same as the liquid temperature, the liquid molecules near the solid wall could form a wetting layer on the wall and this could choke the injector exit so it could be an obstacle against fast jet injection. Therefore, the wetting layer near the solid surface can be eliminated by increasing the wall temperature slightly [8].

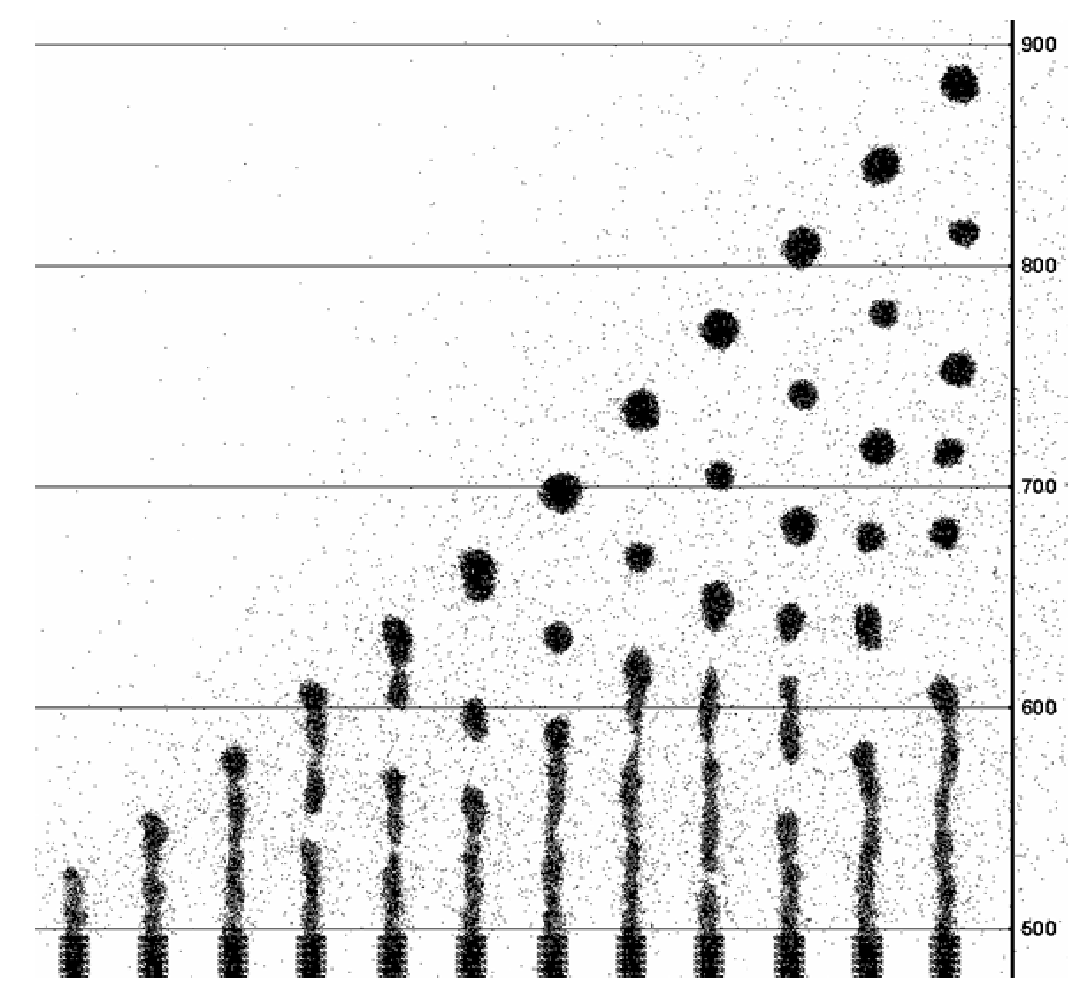

Figure 3. Evolutionary 3-D jet profiles with cylindrical injector. $\left(T_{\text {wall }}^{*}=1.83, T_{\text {liquid }}^{*}=1.50\right)$ Initial plot is at $t^{*}=46.43$ and the time interval between plots is 23.21

Initial axisymmetric disturbances grow quickly and directly lead to the jet head bunching and pinching off. Average intact jet length is less than 100 from the injector tip. The observed droplet diameters before a recombination of drops range from 13 to 18 . The initial diameter of the injector exit is 
8.81 but the observed jet diameter near the injector exit is slightly less than this value. Rayleigh predicted the optimum wavelength of fastest-growing disturbance as 4.51 times the jet diameter [21]. Assuming this disturbance grows and finally leads to the breakup of the jet into droplets, the droplet diameter will be 1.89 times the jet diameter. Rayleigh's prediction about a droplet diameter was reproduced in our results if one takes into account the effect of a slightly decreased liquid jet diameter.

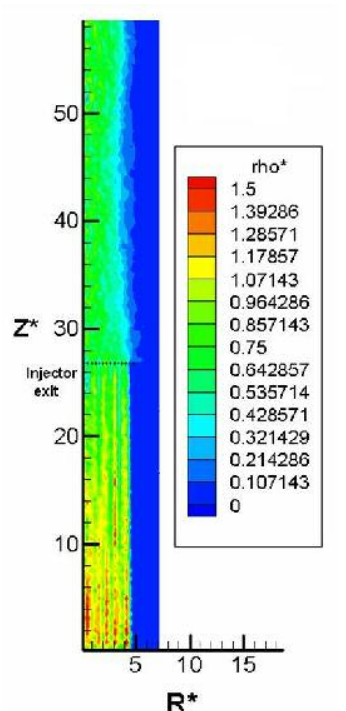

(a) Density Contour

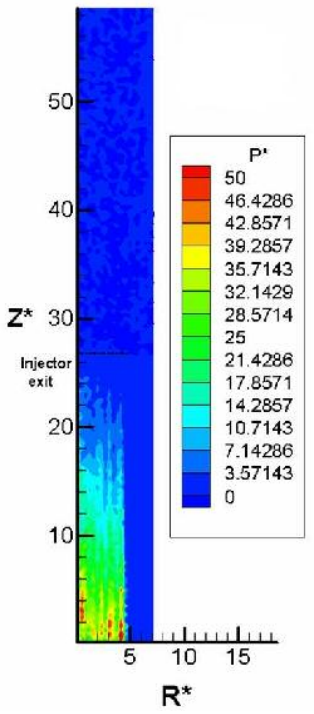

(d) Pressure Contour

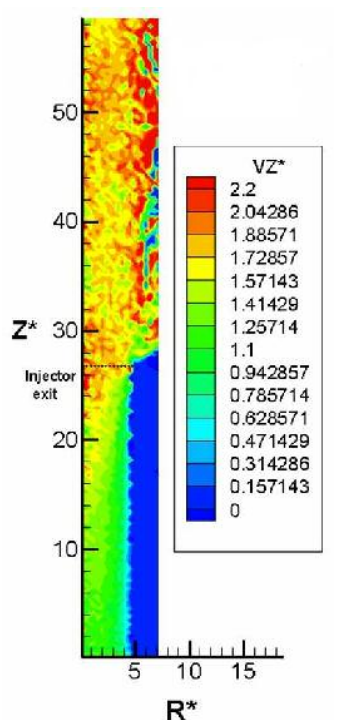

(b) Axial Velocity Contour

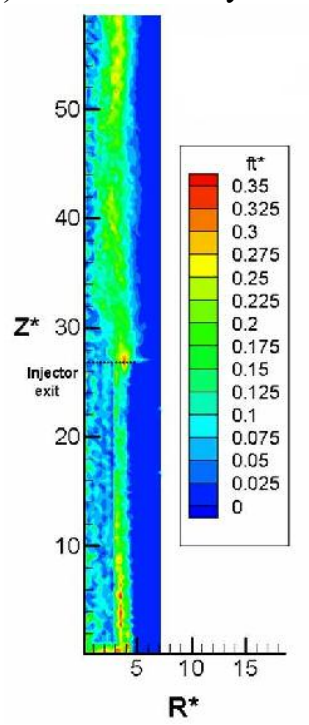

(e) Surface Tension Contour

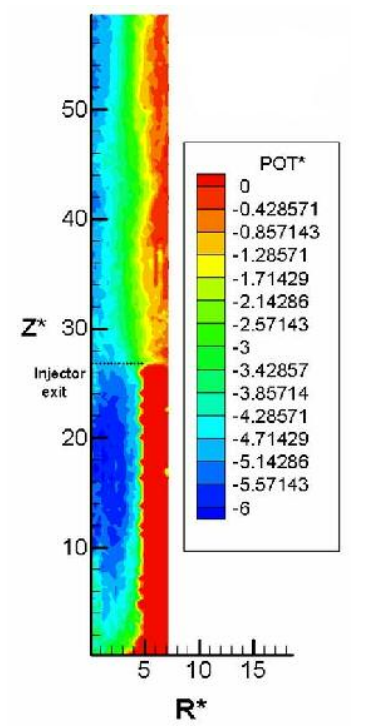

(c) Potential Energy Contour

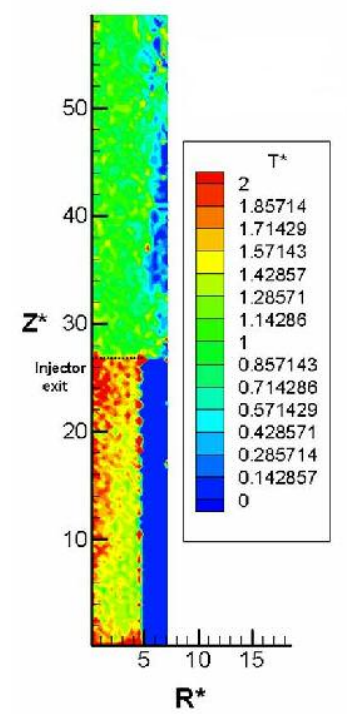

(f) Temperature Contour

Figure 4. 2-D plots of various properties from cylindrical injector jet result. $\left(t^{*}=209\right)$ 


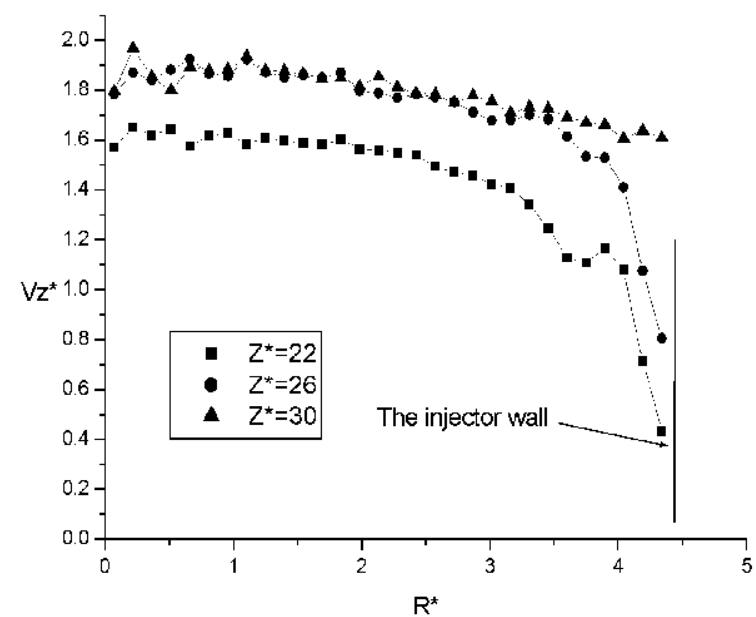

(a) Axial Velocity Profiles

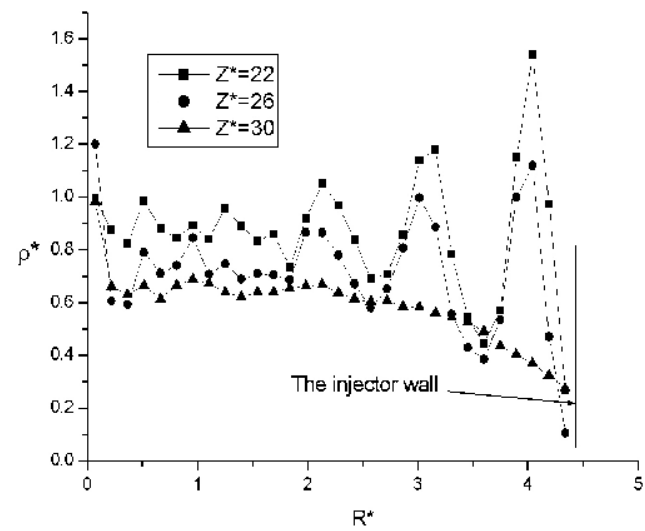

(b) Density Profiles

Figure 5. Axial velocity and density profiles near injector exit.

Using the previously mentioned 2-D plotting technique, various properties of the liquid jet are plotted in figure 4. 2-D sampling was made up to 30 nondimensional distance units past the injector tip $\left(Z^{*} \approx 27\right)$. The properties in figure 4 were sampled from $t^{*}=205$ to $t^{*}=210$.

There are 6 different contour plots. The size of each sampling cell is $0.367 \sigma \times 0.367 \sigma$. With this fine sampling resolution, precise observation of the inner fluid structure is possible. Figure 4-(a) shows the layered density structure of the liquid clearly. This ordered liquid density structure near the solid wall was reported in previous MD studies [19] [22]. The layered density structure is observed extending to the central region of the jet without the decrease of density peak intensity, even at the centre. This strong layered density structure can be regarded as a phase transition to a state with a broken continuous symmetry. Generally this phase transition to a broken symmetry phase was accompanied with rigidity or elastic constant preventing thermal fluctuations from destroying the new state [23]. In addition the axial velocity profile in figure 4-(b) supports the existence of rigidity due to broken symmetry. The development of axial velocity is so weak that the axial velocity is rather uniform across the jet in the region where $Z^{*} \leq 20$. This region also overlaps the region with strong layered density structure.

This layered density structure is attenuated suddenly where $Z^{*} \geq 20$. At this position $\left(Z^{*} \approx 20\right)$, measured pressure, axial velocity and potential energy also experience sudden changes. Pressure drops rapidly as the jet moves near the open end. After this point, a flat axial velocity profile begins to develop into a parabolic profile with strong acceleration in the central region of the jet (see figure 4-(a)). Additionally a sudden increase of average local potential energy (figure 4-(c)) was observed at this point. The definition of the average potential energy is the average amount of the potential energy per atom at a 
given point. This non-continuous jump of potential energy at this point can be regarded as an occurrence of the reverse transition from broken symmetry phase to symmetry phase. With the increase of potential energy, the layered density structure (broken symmetry phase) is also changed into a uniform density structure (symmetry phase). To compensate for the increase of potential energy near the injector exit, the level of kinetic energy (temperature) is slightly decreased after this transition point. This energy conversion process is shown in both the potential energy and temperature contour plots.

It is well known that a solid atomic wall can reproduce the no-slip condition at the solid-liquid interface unless the applied shear rate $\left(\partial V_{Z} / \partial Z\right)$ is excessive [19]. To verify the occurrence of molecular slip in our results, the axial velocity and density profiles at various points near the injector exit are plotted in figure 5. Current injection velocity can cause the shear rate to be in the range of $2-3$ or higher. With this magnitude of shear, molecular slip at the wall cannot be avoided [19]. Axial velocity profiles in figure 5-(a) show the molecular slip at the solid wall clearly. When the jet leaves the injector region $\left(Z^{*}=30\right)$, both density and velocity profiles change rapidly. The fast velocity relaxation also causes a flat axial velocity profile at $Z^{*}=30$ where the distance from the injector exit is only $3 \sigma$. The formation of surface tension is clearly observed in figure 4-(e). As the jet moves to the exit of the injector, the layered density structure is also attenuated from the central region and finally a continuous parabolic profile which indicates the formation of a surface region appears at $Z^{*}=30$. For the measurement of surface tension, we used the method in reference [24], which collects only the attractive intermolecular forces of molecular pairs. The magnitude of measured surface tension is comparable with that from bulk liquid argon at low pressure.

\subsection{Convergent Injector}

A convergent injector with a 45 degree converging half angle was also simulated. Temperature and pressure were calculated inside the solid injector. The temporal jet disintegration process is illustrated in figure 6. The first transient plot is plotted after 69.64 nondimensional times. In spite of a different injector shape, the overall jet breakup is similar to the results with the cylindrical injector. The size of the droplets corresponds to that from Rayleigh theory. Figure 7 shows various 2-D plots from the convergent injector simulation. The axial velocity at the injector tip is slightly higher than that of the cylindrical injector. Because of an inclined injector exit shape, the shape of the density layers was parallel to the solid surface of the injector. However, this layered density structure becomes attenuated in the centre due to a larger injector diameter so the effect of a solid wall is not strong enough to form a density layer structure at the centre. A large stagnant flow region at the injector corner is shown in figure 7-(b). For common engineering scale injectors, there should be a strong recirculation flow at the corner. However, for the 
current nanosized injector case, strong wall adhesive force and surface friction prevent the formation of a recirculation flow. On the contrary, an accelerating flow region is located along the centerline of the jet.

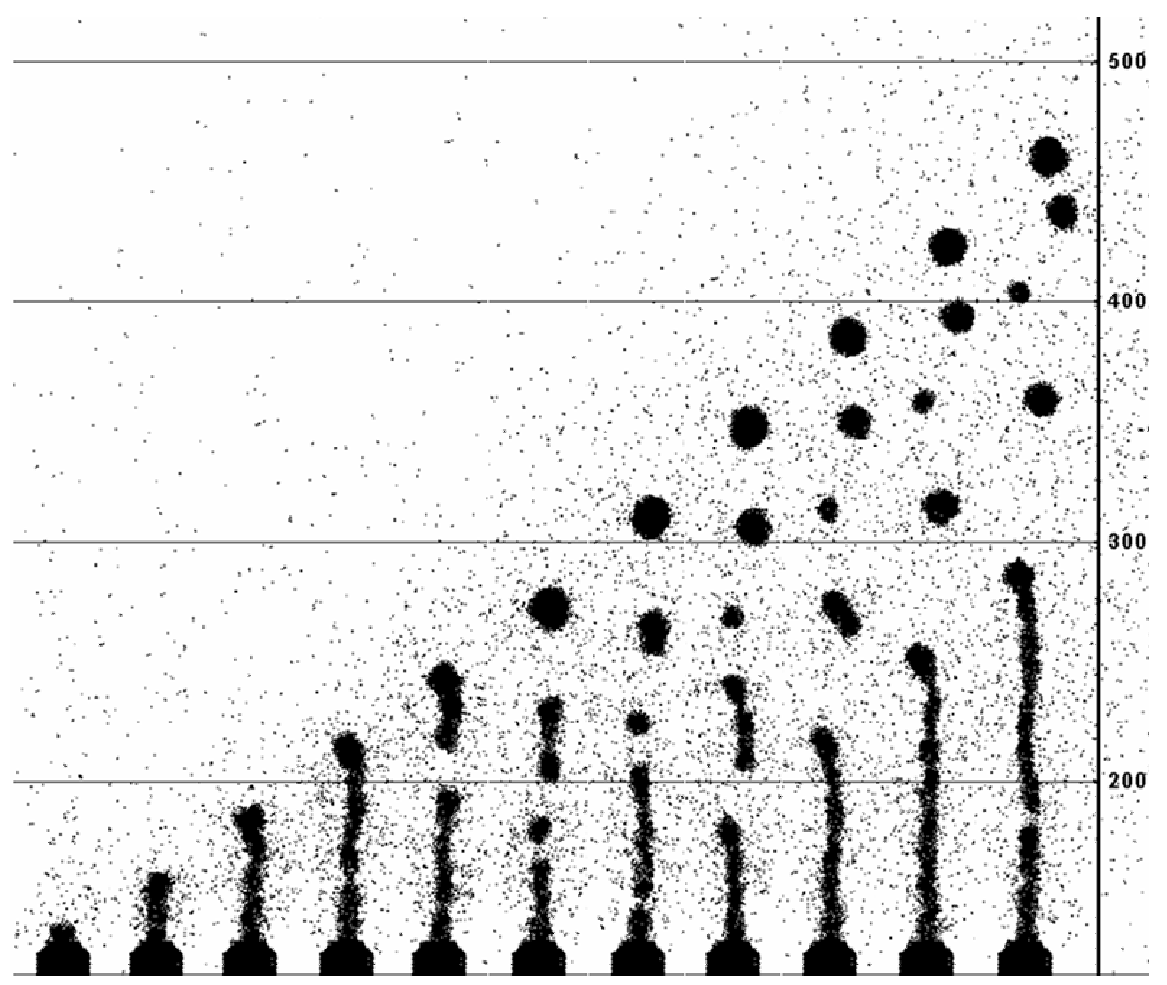

Figure 6. Evolutionary 3-D jet profiles with 45 degree convergent injector. $\left(\mathrm{T}_{\text {wall }}=1.83, \mathrm{~T}_{\text {liq }}=1.45\right)$ Initial plot is at $\mathrm{t}^{*}=69.64$ and the time interval between plots is 23.21

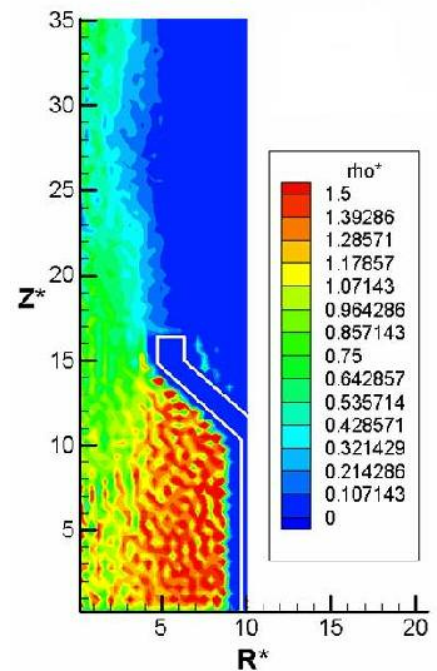

(a) Density Contour

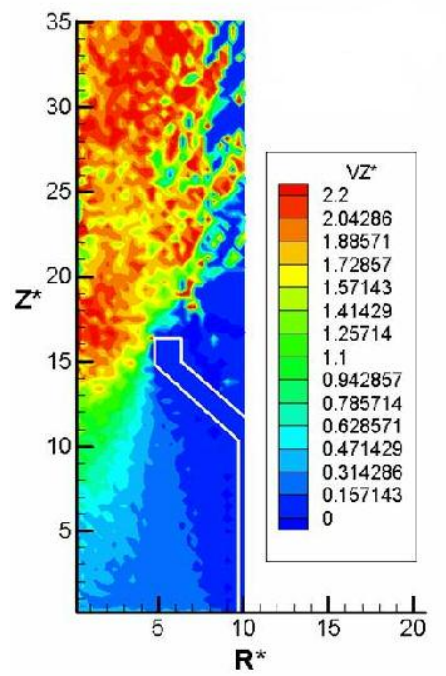

(b) Axial Velocity Contour

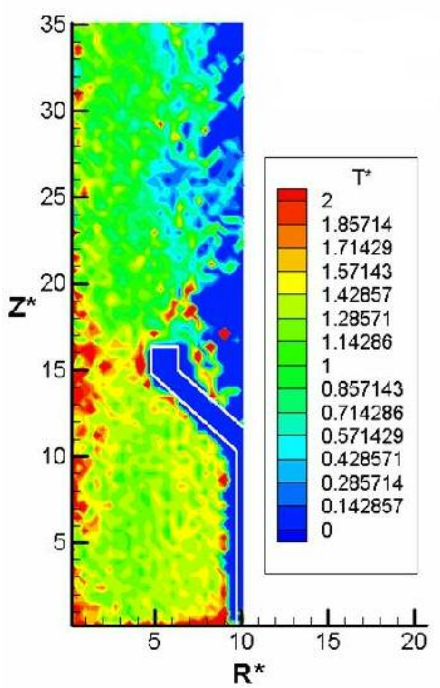

(c) Temperature Contour

Figure 7. 2-D plots of various properties from 45 degree convergent injector jet results. $\left(t^{*}=209\right)$ 


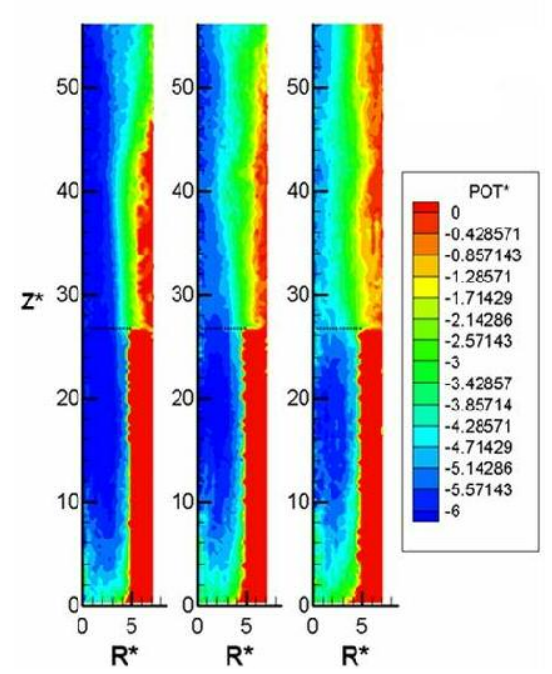

(a) Potential Energy Contours

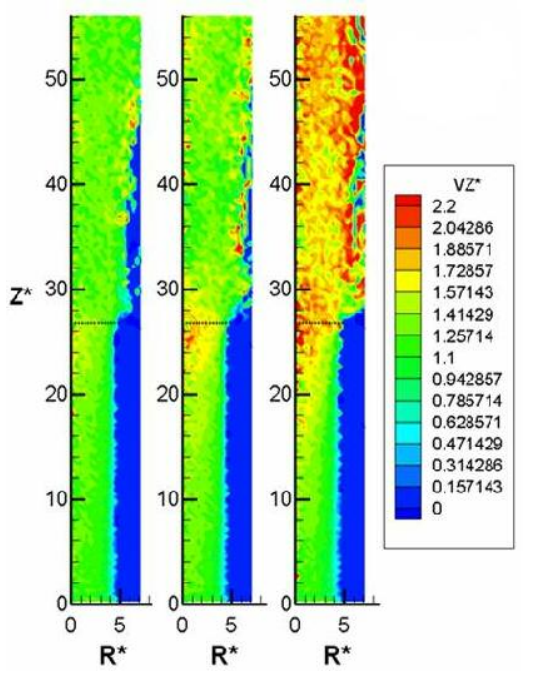

(c) Axial Velocity Contours

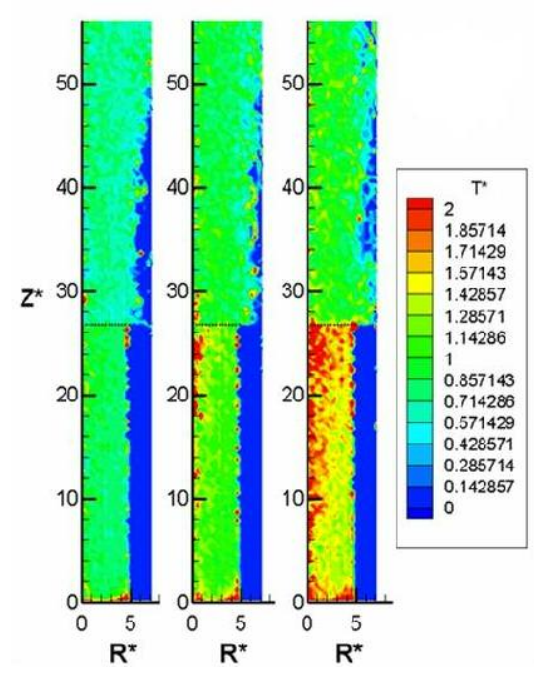

(b) Temperature Contours

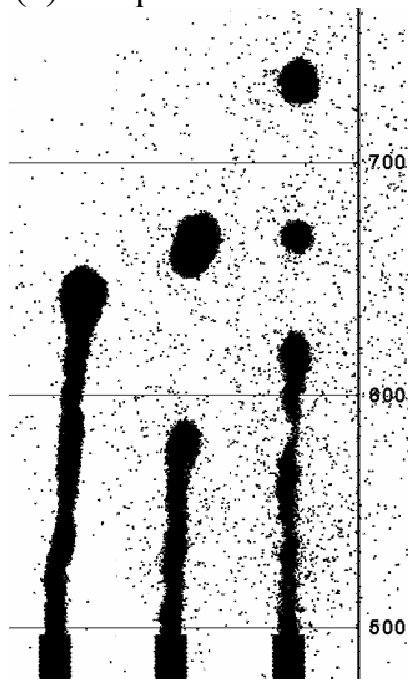

(d) Molecular Scatter Plots

Figure 8. 2-D contour and 3-D scatter plots from different temperature conditions. Simulated liquid temperatures are 0.83 (left), 1.17 (middle) and 1.50 (right) respectively.

\subsection{Temperature dependency of jet breakup behaviour}

A series of simulations with different liquid and wall temperatures were conducted to examine the effect of temperature on jet breakup behaviour. Three different temperatures were simulated with the cylindrical injector, 0.83, 1.17 and 1.50. 2-D and 3-D plots are shown in figure 8. The solid temperature was set to be higher than its liquid temperature by 0.33 in all simulated cases. In the case of the lowest temperature condition $\left(T_{\text {liquid }}^{*}=0.83\right.$ ), a strong negative potential energy region at the jet core lasts even after injection and the slowest injection velocity resulted. The slow growth of surface disturbances was observed in the low temperature results. Thus subsequent jet breakup was delayed and only one droplet 
was observed until the end of the simulation $\left(t^{*}=306\right)$. Vaporized argon atoms were hardly detected in this case. When the liquid temperature is increased, a strong surface instability with enhanced level of vaporization emerges.

In all simulated cases, non-continuous temperature jumps at the injector exit were observed and are shown in figure 8-(b). As discussed in the previous chapter, this energy conversion process results in a reverse phase transition from broken symmetry phase to symmetry phase. However, the amount of additional kinetic energy for this energy conversion process is not enough when the temperature is low. Thus, the level of potential energy of the injected jet is lower than that from higher temperature conditions. This imperfect energy conversion process leads to an incomplete reverse transition so that the rigidity or elasticity, which is a general characteristic of a broken symmetry phase, still remains in the injected jet. The existence of rigidity and elasticity cause a slow jet breakup with less vaporized atoms.

Therefore we concluded that a strong temperature dependency on nanojet breakup behaviour exists and additional kinetic energy is essential for the fast nanojet breakup. However, this phase transition is strongly dependent upon the diameter of the nanosize injector. Much larger injector diameters could prevent the formation of broken symmetry phase.

\section{Concluding Remarks}

With the non-equilibrium molecular dynamics method, the injection of liquid argon into a vacuum environment was simulated. Predicted droplet sizes agree with the predicted values from Rayleigh analysis. Both the cylindrical injector and the 45 degree half angle convergent injector were simulated to examine the effect of injector geometry, however remarkable differences were not observed between the two simulated cases.

A strong cohesive attraction from the solid wall forms the layered density structure parallel to the solid surface. This layered density structure was observed in the entire jet region even at the centre. This can be regarded as a phase transition to a broken symmetry phase. But, near the injector exit, a reverse phase transition to a symmetry phase occurs with an energy conversion process. However, this reverse phase transition was only able to occur when the temperature is high enough to increase the level of potential energy of the injected jet. When the argon temperature in the injector is low, rigidity still remains in the injected jet due to the incomplete reverse phase transition. The existence of rigidity causes a slow nanojet breakup with a weak surface instability. Therefore, it is concluded that the temperature of argon inside the solid injector is the most important controlling factor to subsequent nanojet breakup behaviour. 


\section{References}

[1] Rabinovych O, Pedrak R, Rangelow I, Ruehling H and Maniak M 2004 Microelectron. Eng. 73-74 843-6

[2] Prinz A, Prinz V and Seleznev V 2003 Microelectron. Eng. 67-68 782-8

[3] Yi Y and Reitz R $200215^{\text {th }}$ Annual Conf. on Liquid Atomization and Spray Systems (Madison, WI)

[4] Cheong B and Howes T 2004 Chem. Eng. Sci. 59 2145-57

[5] Sansom M and Biggin P 2001 Nature 414

[6] Koga K, Gao G, Tanaka H and Zeng X 2001 Nature 412 802-5

[7] Maniwa Y, Kataura H, Abe M, Udaka A, Suzuki S, Achiba Y, Kira H, Matsuda K, Kadowaki H and Okabe Y 2005 Chem. Phys. Lett. 401 534-8

[8] Moseler M and Landman U 2000 Science 289

[9] Micci M, Oechsle S and Mayer W $20008^{\text {th }}$ Int. Conf. on Liquid Atomization and Spray Systems (Pasadena, CA. USA)

[10] Ludwig K 2004 Master thesis Pennsylvania State University

[11] Papageorgiou D and Orellana O 1998 SIAM J. Appl. Math. 59 286-317

[12] Reitz R 1987 Atomisation and Spray Tech. 3 307-37

[13] Allen M and Tildesley D 1987 Computer Simulation of Liquids (Oxford University Press)

[14] Yi P, Poulikakos D, Walther J and Yadigaroglu G 2002 Int. J. Heat Mass Transf. 45 2097-100

[15] Plimpton S 1995 J. Comp. Phys. 117 1-19

[16] Koplik J and Banavar J 1995 Phys. Fluids 7

[17] Travis K, Todd B and Evans D 1997 Physical Review E 55(4)

[18] Jabbarzadeh A, Atkinson J and Tanner R 2002 Tribology Int. 35 35-46

[19] Jabbarzadeh A, Atkinson J and Tanner R 1997 J. Non-newtonian Fluid 69 169-193

[20] Tsai D 1979 J. Chem. Phys. 70(3)

[21] Lefebvre A 1989 Atomization and Sprays (Taylor \& Francis)

[22] Travis K and Gubbins K 2000 J. Chem. Phys. 112(4) 1984-94

[23] Chaikin P and Lubensky T 2000 Principles of Condensed Matter Physics (Cambridge University Press)

[24] Kaltz T, Long L, Micci M and Little J 1998 Combust. Sci. and Tech. 136 279-301 